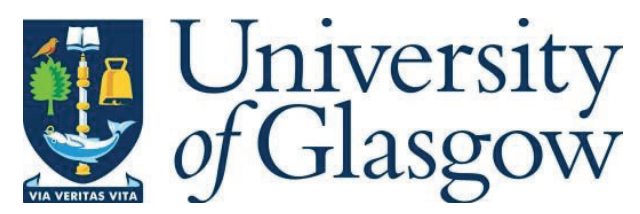

Chang, B., Li, L., Zhao, G., Chen, Z. and Imran, M. A. (2021) Autonomous D2D transmission scheme in URLLC for real-time wireless control systems. IEEE Transactions on Communications, 69(8), pp. 5546-5558.

(doi: 10.1109/TCOMM.2021.3075680).

There may be differences between this version and the published version. You are advised to consult the publisher's version if you wish to cite from it.

http://eprints.gla.ac.uk/239020/

Deposited on: 21 April 2021

Enlighten - Research publications by members of the University of Glasgow http://eprints.gla.ac.uk 


\title{
Autonomous D2D Transmission Scheme in URLLC for Real-Time Wireless Control Systems
}

\author{
Bo Chang, Member, IEEE, Liying Li, Member, IEEE, Guodong Zhao, Senior Member, IEEE, \\ Zhi Chen, Senior Member, IEEE, and Muhammad Ali Imran, Senior Member, IEEE
}

\begin{abstract}
In industrial internet of things (IIoT), ultra-reliable and low-latency communication (URLLC) is proposed to guarantee the requirement of real-time wireless control systems in worst case, so as to maintain the system working in all cases. However, it is extremely challenging to maintain URLLC throughout the whole control process due to the scarcity of wireless resource. This paper develops an autonomous deviceto-device (D2D) communication scheme by jointly considering reliability in URLLC and control requirement. In the proposed scheme, we consider the actual control requirement, i.e., control convergence rate, into communication design, where we find that it can be converted into a constraint on communication reliability. Then, the communication reliability constraint comes from control aspect, instead of URLLC, which leads to that the system does not need to guarantee worst case in URLLC. Second, the sensors autonomously decide whether to be activated with optimal probabilities to participate in the control process, which can maintain the communication reliability requirement with significantly less resource consumption. Simulation results show remarkable performance gain of our method. For instance, compared with fixed activation probability $40 \%$ only considering URLLC, the average power consumption of the proposed method can be reduced by at most about $100 \%$.
\end{abstract}

Index Terms-URLLC, real-time wireless control, autonomous D2D communication.

\section{INTRODUCTION}

$\mathbf{R}$ ECENTLY, real-time wireless control networks are proposed to deal with the limitation in spatial and topology extension and high financial cost in equipment maintenance of the wired control networks for industrial internet of things (IIoT) [1]. In such networks, the control process begins from sensors [2], where the sensors measure the current state of the plants. Then, the controllers estimate the sampled state of the plants and calculate the control inputs. After that, the control inputs are sent to corresponding actuators to update the states of the plants. However, it is very difficult to maintain the high requirement of real-time wireless control. To deal with this issue, ultra-reliable and low-latency communication (URLLC) is adopted as a critical communication scenario in the coming fifth generation (5G) mobile communications [3] [4].

B. Chang and Z. Chen are with the National Key Lab. on Communications, University of Electronic Science and Technology of China (UESTC), Chengdu, 611731, China (e-mail: changb3212@163.com; chenzhi@uestc.edu.cn).

L. Li is with the Department of Mathematics, Physics and Electrical Engineering, Northumbria University, NE1 8ST, UK (e-mail: emma.li@northumbria.ac.uk).

G. Zhao and M. A. Imran are with the School of Engineering, University of Glasgow, Glasgow, G12 8QQ, UK (e-mail: gdngzhao@gmail.com; muhammad.imran@glasgow.ac.uk).

Part of this work was presented in IEEE GLOBECOM 2019
However, it is extremely challenging to maintain both stringent communication reliability and low latency in URLLC by only one transmitter (i.e., one sensor in this paper) to obtain good overall system performance. In this paper, we introduce device-to-device (D2D) communications to deal with this issue since D2D communication is promising to reduce resource consumption, lower communication latency, and improve reliability [5]-[8], where multiple sensors are adopted to serve a specific plant measure the sample parameter, e.g., the temperature of the strip steel in hot rolling process, to achieve the ultra-reliable and low-latency property. Furthermore, the joint design of communication and control are adopted to further reduce the communication resource consumption in URLLC. However, there are two critical challenges to be solved in D2D enabled URLLC for real-time wireless control systems.

The first challenge is how to design D2D activation methods for real-time control applications. The traditional D2D activation methods can be cellular assisted or autonomous [9]-[11]. The cellular assisted methods activate D2D communications with the help of device information at base station (BS), e.g., devices' location. However, the signaling overhead is very high, and the interacting communication with BS leads to high communication latency [12]. Thus, it is not suitable to use cellular assisted D2D activation methods in URLLC for realtime wireless control systems. On the other hand, in traditional autonomous methods, the devices need to transmit reference signals to conduct the D2D activation [13], where the energy consumption is very high and the latency is extremely large. In summary, it is difficult to use existing methods for real-time control applications with URLLC requirements.

The second challenge is how to achieve good overall system performance by jointly considering D2D communication and control systems. URLLC scenario is proposed to guarantee the worst case of mission-critical real-time wireless control. However, the control system may not need such extremely high quality-of-service (QoS) all the time. Thus, wireless resource consumption is expected to be further reduced by jointly designing communication and control [14]. The joint design of communication and control systems is a method by jointly considering parameters from both communication aspect and control aspect to obtain good overall system performance.

There are some recent research on the joint design of control and communication in wireless control systems [15]-[21]. For example, in [15]-[17], the authors took the communication time delay and reliability into control systems and evaluated their effects on the control performance. In [18]-[21], the authors proposed different control algorithms to reduce com- 
munication consumption. However, these research are based on the existing wireless communication protocols, e.g., WiFi or ZigBee, which cannot guarantee the QoS requirement for real-time wireless control systems since the delay is very high [22]. Some other works discussed the joint design of communication and control in URLLC for real-time wireless control systems [2], [23], [24]. For instance, in [23], the authors discussed the effect of different communication QoS on control performance throughout the control process, where they found that the communication energy consumption can be significantly reduced by dynamic QoS allocation while maintaining good control performance. These works show that good overall system performance can be obtained by jointly designing communication and control in URLLC enabled real-time wireless control systems. However, all of them are centralized control via BS, where the stringent QoS in URLLC may not be guaranteed due to high information exchange.

Some research further discussed the joint design of control and communication for vehicular platoon in vehicle-toeverything (V2X) and swarming in UAV networks [25][26]. In these works, the authors discussed the relationship between control stability and communication delay, and developed some methods based on the relationship. However, they focused on group property, e.g., platooning or swarming, which can not be used in real-time wireless control for IIoT. The requirement of each plant in IIoT should be guaranteed in realtime wireless control systems, which can not be maintained by the group property in these related works. In addition, according to [27], considering each specific plant, the system stability is affected by the communication reliability, instead of the transmission time delay. The stability is guaranteed by the control convergence rate constraint in this paper. Based on that, we analyze the relationship between the control convergence rate and communication reliability and discuss the effect of the control convergence rate on the communication consumption. In addition, since it is required that communication should maintain ultra-reliability and low-latency property in realtime wireless control for each plant, finite blocklength and its corresponding channel capacity is adopted, which is novel for wireless communications and is not discussed in these related works. Finally, the motivation of this paper is to match sensors with each plant to maintain stringent communication and control requirements, which is extremely challenging and not discussed in these works.

We notice that some research discussed the D2D design in URLLC for industrial automation [28] [29], where they tried to maintain the extremely high QoS requirement in URLLC. For instance, the authors in [28] developed a twophase transmission protocol to exploit the D2D transmission from the controller to the actuator for URLLC. Under their protocol, each group's messages are combined together and multicast to the leaders from the BS in the first phase, while the leaders relay the messages to the other users in their groups in the second phase. However, the transmitter selection in D2D communications is BS assisted, where the intercommunication time delay cannot be ignored. In addition, the communication from BS to the final devices is divided into two phases, which raises the transmission time delay. These transmission settings would significantly increase the resource consumption to maintain the time delay and reliability requirements in URLLC. Furthermore, the authors missed the actual control requirement and only the high reliability from URLLC was guaranteed, where the huge amount of resource consumption in URLLC cannot be reduced. In summary, the D2D design in these works is to guarantee the QoS URLLC at all costs. This may impede the deployment of URLLC in IIoT due to the serious scarcity of wireless resource, instead of reducing the resource consumption to benefit the URLLC deployment.

This paper proposes a new autonomous D2D transmission scheme to deal with the extremely high QoS requirements in URLLC for real-time wireless control systems. In [8], we proposed the primary idea of autonomous D2D transmission scheme without control requirement. In this paper, our goal is to minimize D2D transmission energy consumption by jointly considering reliability constraint in URLLC and control constraint, where the allocated time resource of URLLC is assumed to be constant in this paper to simplify our discussion. In particular, we answer the questions on how to activate D2D transmission and how to meet control requirements. The main contributions of this paper are as follows.

- We propose a probability-based D2D activation and power allocation scheme, which allows each sensor autonomously to decide whether to participate the control process without interactive communications. This scheme can guarantee the communication reliability requirement with time constraint and the control requirement.

- We analyze the relationship between control and communication, where we find that the control convergence rate requirement can be converted into the communication reliability constraint. This provides a guidance on D2D power allocation, and can significantly reduce energy consumption.

- We develop an optimal D2D transmission method by balancing failed reduction efficiency (FRE) for all sensors, which can optimize activation probability and minimize transmission power under the reliability constraint in wireless communications while guaranteeing control requirement. Here, the FRE is defined as the ratio of the failed transmission probability and the corresponding transmission energy consumption.

The rest of this paper is organized as follows. In Section II, the system model is presented. In Section III, the optimization problem with both communication and control constraints is formulated. In Section IV, we obtain the optimal probabilitybased activation method and transmission power allocation method. In Section V, simulation results are provided to show the performance. Finally, Section VI concludes the paper.

\section{SySTEM MODEL}

As shown in Fig. 1, we consider a wireless control model that $M$ independent sensors (i.e., transmitting devices) intend to serve a plant (i.e., receiving device) by direct D2D communications. However, the proposed method can be easily extended to the scenario with $K$ plants. When the plant needs to work, it would first send a request to the $\mathrm{BS}$ in $5 \mathrm{G}$ 


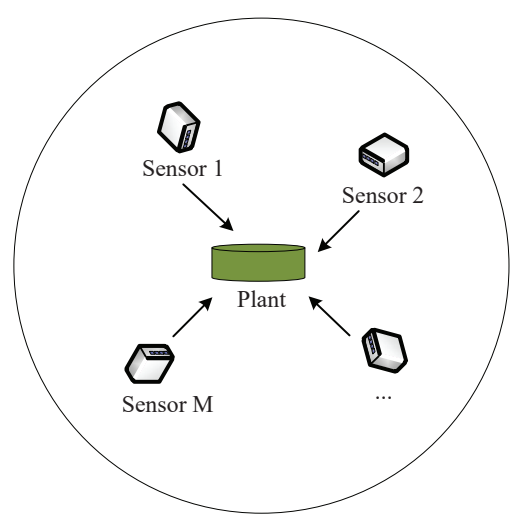

(a) communication subsystem

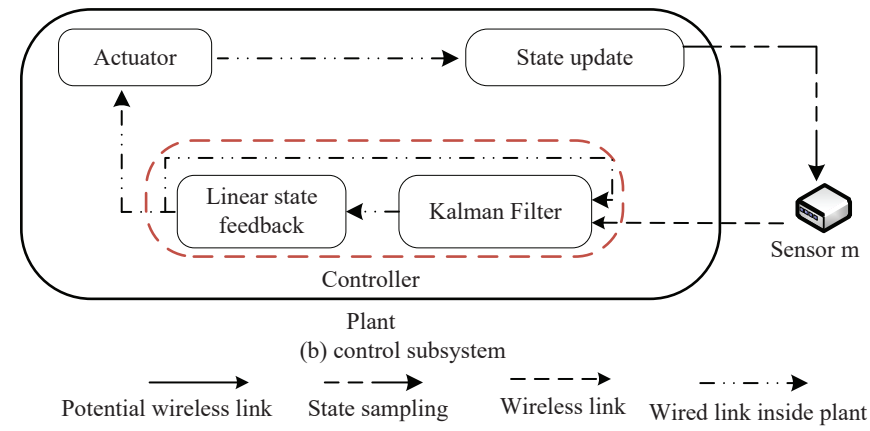

Fig. 1: System model.

URLLC scenario. Then, the BS would broadcast the necessary information, i.e., the position of the plant, the operation time of the plant, and the allocated frequency, to the sensors. Then, the sensors would be activated and transmit using the proposed method. Furthermore, if a sensor malfunction is detected, the BS can set the activation probability of this sensor to be zero via the broadcasting phase. In such a system, each sensor is activated with a certain probability, where the active sensors sample the plant state and transmits it to the controller embedded in the plant via the wireless channel. Then, the controller chooses the strongest signal as its desired signal, calculates the control command, and sends a command to the actuator to update plant's current state ${ }^{1}$.

The system model in Fig. 1 is widely considered in real industrial scenarios [31], we provide a real industrial application by the following Example 1.

Example 1: As shown in Fig. 2, a typical industrial scenario, i.e., hot rolling process in a factory, is adopted to show that the adopted model is valid. Such a scenario contains heating furnaces, reversing rougher, finishing mill, laminar cooling, and down cooling [31], where the distance between different sub-process is given in Fig. 2. According to [32], the area of the factory building is about 150000 square meters of floor space, which is scarcely partitioned. Ceiling height varies from 12.5 to $18 \mathrm{~m}$. The roof is formed from tar paper and gravel and is supported by sheet steel and dense steel truss work. To achieve better communication performance, the height of wireless communication facilities (e.g., the receiver wired

\footnotetext{
${ }^{1}$ Note that the sensors obtain state observations and then send the observations to the controller for state estimation. [30].
}

connected to the plant and its corresponding sensors) is set to $2 \mathrm{~m}$ above the floor [32]. Clusters consisting of different wireless communication facilities are divided by the served plants. For instance, the receiver connected to the furnaces and the corresponding sensors are divided into cluster 1 . In such a large area scarcely partitioned with plenty of plants and the height of wireless communication facilities over the floor, both the free space channel gain model and Rayleigh fading are valid according to the measurement in [32]. To avoid interference, different frequency bands are allocated for different clusters, and orthogonal frequency division multiple access (OFDMA) is adopted to avoid interference among different sensors in each cluster. As shown in Fig. 2, the state, i.e., the temperature of the strip steel in each sub-process, is monitored by massively deployed sensors ${ }^{2}$. Compared with only using one sensor in a sensor-plant loop, multiple sensors can maintain ultra-reliable and low-latency requirement in real-time control. Then, the measured parameter is sent to the controller over wireless channels. After that, the state estimation is calculated by the Kalman Filter inside the controller, and the control decision is made by the linear feedback unit. Finally, the decision is sent to the actuator, where the valves of water nozzles is adjusted to reach the required exit temperature of the strip steel.

In the following of this section, the system model considering both communication latency and reliability is presented for the performance evaluation in real-time wireless control systems. Note that all the notations to be used throughout the paper for communication and control are summarized in Table I and II, respectively.

\section{A. Communication Model}

In this subsection, we provide a typical communication model with transmission latency and packet error probability in URLLC. As shown in Fig. 1 (a), to guarantee the fairness of each plant, $M$ sensors are uniformly distributed ${ }^{3}$ in a certain circle region with radius $R$, and the plant is in the center of the region. Here, each sensor is activated based on a certain probability. In addition, the plant only treats the strongest signal from the sensors as its desired signal and ignores others. Furthermore, OFDMA is adopted to avoid interference. In the following of this subsection, we introduce the channel model

\footnotetext{
${ }^{2}$ In practice, it is very risky to deploy one or two sensors that are close to the controller. This is because line-of-sight communications can not always be guaranteed in practical setup with many uncontrollable factors. In other words, the sensors with short distance do not always provide strong signals, while some sensors with larger distance may provide strong signals in some situations. Another reason that we recommend deploying a large number of sensors over the whole area is to meet the required performance in URLLC.

${ }^{3}$ In this paper, we assume that the sensors are uniformly distributed in the given area. This is because the future factories are expected to face the dynamic market demand, short product life-cycle, and flexibility need, where re-configurable manufacturing system will be one of the most important solutions. In such a system, manufacturing facilities are with great flexibility to form a new production line for customized products. Then, it becomes a natural choice to deploy sensors with uniform distribution, which would maximize the flexibility of the reconfigurable manufacturing [33]. In addition, other distributions would work well in some cases or applications. In this paper, the proposed method also works in these cases by changing the uniform distribution to other distributions.
} 


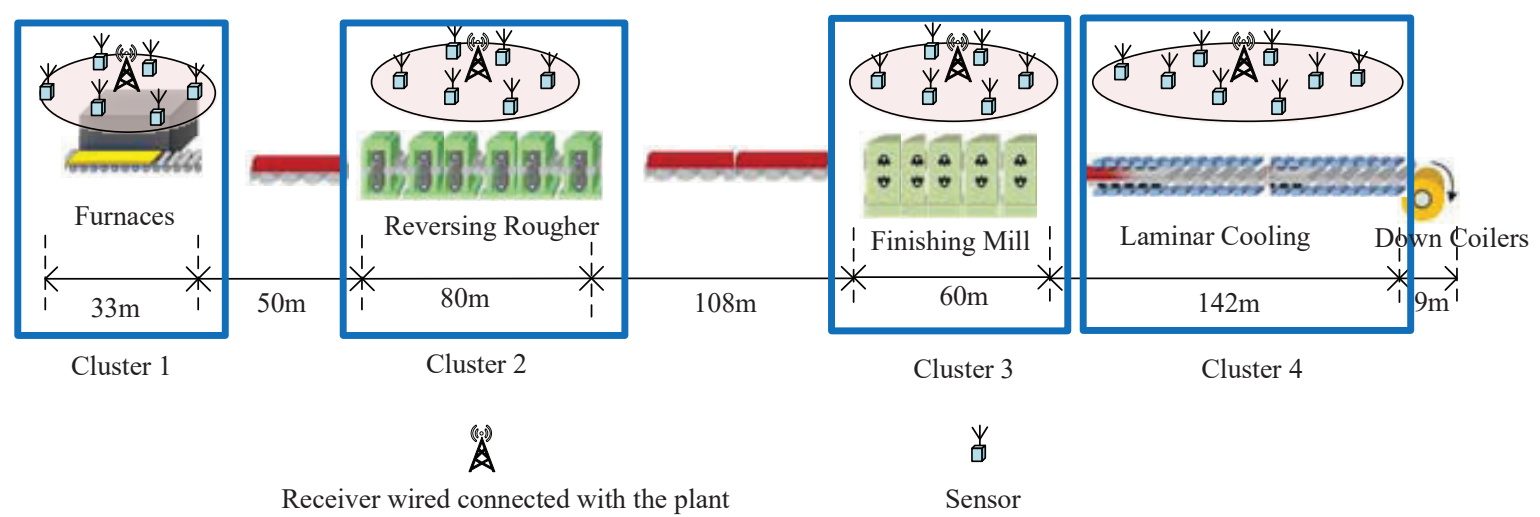

Fig. 2: A typical industrial scenario of the system model: hot rolling process.

TABLE I: Summary of Notations for Communication

\begin{tabular}{|c|c|c|c|}
\hline$B_{m}$ & Allocated bandwidth for the $m$-th sensor & $R$ & Coverage radius of the sensor distribution \\
\hline$C_{m}$ & Shannon capacity of the $m$-th sensor $n$ & $\alpha_{n}$ & Indicator for packet loss \\
\hline$g_{m}$ & Path-loss of the $m$-th sensor & $T_{u}$ & Time resource of the uplink for the $m$-th sensor \\
\hline$h_{m}$ & Small scale fading for the $m$-th sensor & $V_{m}$ & Channel dispersion for the $m$-th sensor \\
\hline$l_{m}$ & Distance between the $m$-th sensor and the plant & $\gamma_{m}$ & Received SNR from the $m$-th sensor \\
\hline$N_{0}$ & Variance of the AWGN at the plant & $p_{\max }$ & Maximum of transmission power \\
\hline$M$ & Total number of sensors in the coverage of the base station & $\varepsilon$ & Packet loss probability of the uplink for the plant \\
\hline$m$ & Index of the sensors $(1 \leq m \leq M)$ & $\varepsilon_{t h}$ & Maximum packet error probability in communications \\
\hline$p_{m}$ & Transmission power spectral density & $\gamma_{t h}$ & SNR threshold \\
\hline$a$ & The inside radius of a typical circle ring & $b$ & The outside radius of a typical circle ring \\
\hline $2 r$ & The distance between the inside bound to outside bound & $\varepsilon_{0}$ & Normalization of the transmission error \\
\hline$J_{c}(a)$ & The average energy consumption & $M_{a}$ & The number of sensors inside the circle ring \\
\hline$\varphi$ & Normalization of the sensor's active threshold & $\varphi_{m}$ & The active threshold for the $m$-th sensor \\
\hline
\end{tabular}

used and the channel capacity between sensor $m$ and the plant in URLLC, respectively.

1) Channel Model: We consider that the channel model consists of the small-scale fading and large-scale attenuation coefficients between sensor $m$ and the plant, which are represented as $h_{m}$ and $g_{m}$ for the uplink from the $m$-th sensor to the plant, respectively. We assume that the large-scale attenuation coefficient is represented by path-loss, which can be expressed as [34]

$$
g_{m}=\frac{C}{l_{m}^{\lambda}},
$$

where $l_{m}$ is the distance between transceiver, $C$ is a constant, and $\lambda \in[2,6]$ is the path loss factor. Since we consider $M$ sensors are uniformly distributed in a circle with the plant in the center, then we have the probability distribution function (PDF) of the sensors with distance $l$ as

$$
f_{l}(l)=\frac{2 l}{R^{2}}
$$

where $R$ is the radius of the circle. Then, we can obtain the pdf of the path loss as

$$
f_{g_{m}}\left(g_{m}\right)=\frac{C g_{m}^{-2}}{R^{2}}, \quad g_{m} \geq \frac{C}{R^{2}},
$$

where $\lambda=2$ is adopted [32].

The small-scale fading $h_{m}$ follows Rayleigh distribution with mean zero and variance $\sigma_{0}^{2}=1$ [32]. Then, the PDF of its power can be expressed as

$$
f_{h_{m}^{2}}\left(h_{m}^{2}\right)=e^{-h_{m}^{2}}
$$

However, the end-to-end (E2E) latency is no more than $1 \mathrm{~ms}$ in URLLC, which is less than the channel coherence time [35]. Then, for the $m$-th sensor, the small-scale fading is constant during the transmission period [36].

2) Channel Capacity: According to [35] and [37], we can obtain the successful transmit bits in one frame for the $m$-th sensor in URLLC can be expressed as

$$
R_{m}=C_{m}-\sqrt{\frac{V_{m}}{T_{u} B_{m}}} f_{Q}^{-1}\left(\varepsilon_{m}\right)+\frac{\log \left(T_{u} B_{m}\right)}{2 T_{u} B_{m}},
$$

where the first term on the right hand of (5) is the achievable Shannon capacity without transmission error, the second term 
TABLE II: Summary of Notations for Control

\begin{tabular}{|c|c|c|c|}
\hline $\mathbf{A}$ & System parameter on state in continuous control function & $\mathbf{u}(t)$ & Control command \\
\hline B & System parameter on command in continuous control function & $c$ & Control performance constraint on wireless communications \\
\hline $\mathbf{x}(t)$ & Plant state in continuous control function & $\mathbf{u}_{n}$ & Control command for simplify \\
\hline$N$ & Total sampling time index in control process & $\mathbf{x}_{n}$ & Plant state in discrete time control function \\
\hline$n$ & Sample time index & $\Delta(\cdot)$ & Lyapunov-like function \\
\hline $\mathbf{n}(t)$ & Disturbance caused by AWGN in continuous control function & $\boldsymbol{\Phi}_{0}^{n}$ & System parameter on input in discrete control function \\
\hline $\mathbf{n}_{n}$ & Disturbance in discrete time control function & $\boldsymbol{\Phi}_{1}^{n}$ & System parameter on input caused by time delay \\
\hline$\overline{\mathbf{n}}_{n}$ & Generalized disturbance & Q & Given positive definite matrix \\
\hline $\mathbf{R}_{m}$ & Variance of $\mathbf{n}_{m}(t)$ & $\mathbf{\Omega}_{d}$ & Generalized system parameter on state \\
\hline$s_{n}$ & Sample period of the $m$-th plant at time index $n$ & $\mathbf{\Omega}_{n}$ & System parameter on state in discrete control function \\
\hline $\bar{s}_{n}$ & Idle time before the sampling at time index $n$ & $\xi_{n}$ & Generalized plant state \\
\hline $\mathbf{R}_{m}$ & Variance of $\overline{\mathbf{n}}_{n}$ & $\rho$ & Control convergence rate \\
\hline
\end{tabular}

is the minus error bits introduced by channel dispersion $V_{m}$, and the third term is the approximation of the reminder terms of order $\log \left(T_{u} B_{m}\right) /\left(T_{u} B_{m}\right)$. In addition, $B_{m}$ is the occupied bandwidth, $\varepsilon_{m}$ is the transmission error probability, and $f_{Q}^{-1}(\cdot)$ is inverse of $Q$ function. We need to note that $T_{u}$ is the allocated time resource, which is treated as the transmission time delay ${ }^{4}$. Furthermore, we assume that the single-sided noise spectral density is represented by $N_{0}$, then according to [35], we have Shannon capacity $C_{m}$ and channel dispersion $V_{m}$ as follows, respectively,

$$
C_{m}=T_{u} B_{m} \log \left(1+\gamma_{m}\right),
$$

and

$$
V_{m}=T_{u} B_{m}(\log e)^{2}\left(1-\frac{1}{\left(1+\gamma_{m}^{2}\right)}\right),
$$

where $\gamma_{m}$ is the received signal-to-noise-ratio (SNR) and can be expressed as

$$
\gamma_{m}=\frac{h_{m}^{2} B_{m} g_{m} P_{m}}{N_{0} B_{m}}=\frac{h_{m}^{2} g_{m} p_{m}}{N_{0}},
$$

where $p_{m}$ is the single-sided transmission power of the $m$-th sensor.

\section{B. Control Model}

In this subsection, we provide the real-time control model with communication time delay and reliability. As shown in Fig. 1 (b), the control process is conducted as follows. First, $M$ sensors are activated with a certain probability to guarantee the control requirement, which take samples of the current plant state and transmit them to the controller inside the plant. Then, the controller estimates the state by Kalman Filter based on the strongest signal among the sensors, calculates the control command, and sends it to the actuator by wired link. Here, the

\footnotetext{
${ }^{4}$ Here, only allocated time resource is considered as the transmission time delay in the transmission. Since we focus on the effect of control requirement on communication design, the serving time delay, queueing time delay, and other time delays are not considered to simplify our discussion, which is treated as our future work.
}

Kalman Filter inside the controller is responsible for the state estimation by both prior information and posterior information, which is proved to be an optimal estimator in linear control process. More details about the calculations in the Kalman Filter can be found in [24]. Finally, the plant state updates by the received control command. Based on the above control process, the linear differential equation ${ }^{5}$ of the plant can be expressed as [16]

$$
d \mathbf{x}(t)=\mathbf{A x}(t) d t+\mathbf{B u}(t) d t+d \mathbf{n}(t)
$$

where $\mathbf{x}(t)$ is the plant state, $\mathbf{u}(t)$ is the control input, and $\mathbf{n}(t)$ is the disturbance caused by additive white gaussian noise (AWGN) with zero mean and variance $\mathbf{R}$. In addition, $\mathbf{A}$ and $\mathbf{B}$ represent the physical system parameter matrices (more details can be obtained in [39]).

We assume that $s_{n}$ represents the sample period at time index $n$, which consists of the wireless transmission time delay $T_{u}$ and an idle period $\bar{s}_{n}$. Their relationship can be expressed as

$$
s_{n}=\bar{s}_{n}+T_{u},
$$

where $n=1,2, \cdots, N$ represents the sampling time index in the control process. Then, the discrete time control model with time delay $T_{u}$ can be obtained as [15]

$$
\mathbf{x}_{n+1}=\boldsymbol{\Omega}_{n} \mathbf{x}_{n}+\boldsymbol{\Phi}_{0}^{n} \mathbf{u}_{n}+\boldsymbol{\Phi}_{1}^{n} \mathbf{u}_{n-1}+\mathbf{n}_{n},
$$

where $\boldsymbol{\Omega}_{n}=e^{\mathbf{A} s_{n}}, \boldsymbol{\Phi}_{0}^{n}=\left(\int_{0}^{\bar{s}_{n}} e^{\mathbf{A} t} d t\right) \cdot \mathbf{B}$, and $\boldsymbol{\Phi}_{1}^{n}=$ $\left(\int_{\bar{s}_{n}}^{s_{n}} e^{\mathbf{A} t} d t\right) \cdot \mathbf{B}$.

Assuming $\xi_{n}=\left(\begin{array}{ll}\mathbf{x}_{n}^{T} & \mathbf{u}_{n-1}^{T}\end{array}\right)^{T}$ is the generalized state, then the control function in (11) can be rewritten as

$$
\xi_{n+1}=\boldsymbol{\Omega}_{d} \xi_{n}+\boldsymbol{\Phi}_{d} \mathbf{u}_{n}+\overline{\mathbf{n}}_{n}
$$

${ }^{5}$ Note that nonlinear systems can be replaced by linear systems with linearization in a small range near the operating point, which is reasonable and widely used [38]. From this perspective, our method can be used in nonlinear models. 
where $\overline{\mathbf{n}}_{n}=\left(\begin{array}{ll}\mathbf{n}_{n}^{T} & 0\end{array}\right)^{T}$ and $\boldsymbol{\Phi}_{d}=\left(\begin{array}{l}\boldsymbol{\Phi}_{0}^{n} \\ \mathbf{I}\end{array}\right)$. We assume $\boldsymbol{\Omega}_{n}=\boldsymbol{\Omega}$. Then, we have $\boldsymbol{\Omega}_{d}=\left(\begin{array}{cc}\boldsymbol{\Omega} & \boldsymbol{\Phi}_{1}^{n} \\ 0 & 0\end{array}\right)$.

Considering the packet loss, we have the successful packet transmission probability $\operatorname{Pr}\left\{\alpha_{n}=1\right\}=1-\varepsilon$ and the failed packet transmission probability $\operatorname{Pr}\left\{\alpha_{n}=0\right\}=\varepsilon$, where $\varepsilon$ represent that $M$ sensors are failed in transmission. In addition, we assume that the state estimator is perfect. Then, we have the close-loop system in (12) can be rewritten as

$$
\xi_{n+1}= \begin{cases}\boldsymbol{\Omega}_{d} \xi_{n}+\boldsymbol{\Phi}_{d} \mathbf{u}_{n}+\overline{\mathbf{n}}_{n}, & \text { if } \alpha_{n}=1, \\ \mathbf{\Omega}_{d} \xi_{n}+\overline{\mathbf{n}}_{n}, & \text { if } \alpha_{n}=0,\end{cases}
$$

which can be rewritten in a general way as

$$
\xi_{m, n+1}= \begin{cases}\boldsymbol{\Omega}_{e_{1}} \xi_{m, n}+\overline{\mathbf{n}}_{n}, & \text { if } \alpha_{n}=1 \\ \boldsymbol{\Omega}_{e_{0}} \xi_{m, n}+\overline{\mathbf{n}}_{n}, & \text { if } \alpha_{n}=0,\end{cases}
$$

where $\boldsymbol{\Omega}_{e_{1}}=\boldsymbol{\Omega}_{d}+\boldsymbol{\Phi}_{d} K, \boldsymbol{\Omega}_{e_{0}}=\boldsymbol{\Omega}_{d}$, and $K$ is the control command feedback parameter.

In the above discussion, we have obtained the wireless control model ${ }^{6}$ where both communication time delay and packet loss have been taken into account. In the following of this paper, we will formulate the optimization problem and propose corresponding iterative method to obtain the D2D transmission scheme.

\section{Problem Formulation for D2D Activation And TRANSMISSION}

In this section, we formulate the D2D activation and power allocation problem to guarantee the stringent QoS while minimizing communication energy consumption. In the following, we first provide objective function, communication constraint, and control constraint. Then, we formulate the optimization problem to minimize total communication energy consumption.

\section{A. Objective Function}

Since sensors are usually powered by battery [41], minimizing energy consumption is very important in real-time wireless control systems. Thus, the objective is to minimize energy consumption $^{7}$ at each time index $n$, which can be expressed as

$$
J_{E}=\mathbb{E}\left[\sum_{m=1}^{M} T_{u} B_{m} p_{m, n}\right] .
$$

In this paper, instead of discussing the tradeoff of the resource consumption in URLLC, we intend to show the performance of the proposed autonomous D2D transmission scheme in URLLC for real-time wireless control systems by jointly considering communication and control aspects, where the adopted criteria is energy consumption. To simplify the

\footnotetext{
${ }^{6}$ According to [40], to maintain the stability of the wireless control system, the following assumption should be satisfied: The packet loss probability in URLLC and the control system parameters satisfy $\rho\left((1-\varepsilon)\left(\boldsymbol{\Omega}_{d}+\boldsymbol{\Phi}_{d} K\right) \otimes\right.$ $\left.\left(\boldsymbol{\Omega}_{d}+\boldsymbol{\Phi}_{d} K\right)+\varepsilon \boldsymbol{\Omega}_{d} \otimes \boldsymbol{\Omega}_{d}\right)$, where $\rho(\cdot)$ is the spectral radius, $K$ is the control command feedback parameter, and $\otimes$ is the Kronecker product.

${ }^{7} \mathrm{Here}$, since we focus on wireless transmission, only communication energy consumption is considered in this paper.
}

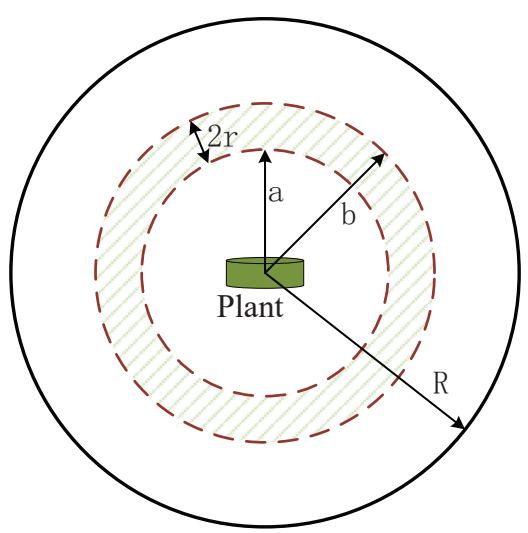

Fig. 3: The model to calculate the reliability probability.

discussion, we assume that the allocated time resource (i.e., the transmission time delay) $T_{u}$ and bandwidth $B_{m}$ are constant. Thus, the energy consumption is equivalent to the power consumption. Then, (15) can be rewritten as

$$
J=\mathbb{E}\left[\sum_{m=1}^{M} p_{m, n}\right],
$$

where the expectation is calculated over the uniform distribution of the sensors, i.e.,the distance of the sensors from the plant.

\section{B. Control Constraint}

We consider the control state convergence rate as the control performance. To obtain the reduction rate, we adopt Lyapunovlike control cost function [2]

$$
\Delta\left(\xi_{n}\right)=\xi_{n}^{T} \mathbf{Q} \xi_{n},
$$

where $\mathbf{Q}$ is positive definite. To guarantee the control stability, the Lyapunov-like function should decrease at given convergence rate $^{8} \rho<1$, which can also guarantee the state return to the pre-set point. Then, for any possible $\xi_{n}$, the Lyapunov-like functions needs to satisfy [42]

$$
\mathbb{E}\left[\Delta\left(\xi_{n+1}\right) \mid \xi_{n}\right] \leq \rho \Delta\left(\xi_{n}\right)+\operatorname{Tr}\left(\mathbf{Q R} \mathbf{R}^{\prime}\right),
$$

where $\mathbb{E}[\cdot]$ represents the expectation operator and $\mathbf{R}^{\prime}=$ $\left(\begin{array}{ll}\mathbf{R}^{T} & 0\end{array}\right)^{T}$ is the variance of $\overline{\mathbf{n}}_{n}=\left(\begin{array}{ll}\mathbf{n}_{n}^{T} & 0\end{array}\right)^{T}$.

\section{Communication Constraint}

The QoS requirements in URLLC include low latency and ultra-reliability. We assume that the latency is no more than the allowed upper bound in this paper. Then, the communication constraint introduced by QoS requirement is the ultrareliability, where we assume that the upper bound of the packet loss probability is $\varepsilon_{t h}$. Here, the packet loss probability for the $m$-th sensor consists of two parts: the first part is the packet error probability $\varepsilon_{m}$ in (5), and the second part is the packet loss probability when SNR $\gamma_{m}$ is less than a threshold $\gamma_{t h}$ that can guarantee the received bits. To calculate the overall

\footnotetext{
${ }^{8}$ It is shown in [42] that the control convergence is guaranteed with $\rho<1$.
} 
reliability probability inside the circle with radius $R$, we divide it into multiple circle rings. As shown in Fig. 3, we consider a typical circle ring with inside radius $a$, outside radius $b$ and the length from the inside bound to outside bound $2 r$. Then, the number of sensors inside the circle ring can be expressed as

$$
M_{a}=\frac{b^{2}-a^{2}}{R^{2}} \cdot M,
$$

Furthermore, we assume that the activated probability of the sensors insider the circle ring is represented by $P_{a}(a)$ and the transmission power of these sensors is represented by $p(a)$ when $r \rightarrow 0$. Then, we can obtain that the cumulative distribution function (CDF) of the SNR of the sensors inside the circle ring can be expressed as

$$
\begin{aligned}
& F_{\Gamma}(\gamma \mid a \leq l \leq b)=\operatorname{Pr}\left\{\frac{h^{2} g p(l)}{N_{0}} \leq \gamma\right\} \\
& =\int_{\frac{C}{b^{2}}}^{\frac{C}{a^{2}}} \int_{0}^{\frac{\gamma N_{0}}{p(a) g}} f_{g}(g) f_{h^{2}}\left(h^{2}\right) \mathrm{d} g \mathrm{~d} h^{2} \\
& =\int_{\frac{C}{b^{2}}}^{\frac{C}{a^{2}}} \int_{0}^{\frac{\gamma N_{0}}{p(a) g}} \frac{C g^{-2}}{R^{2}} e^{-h^{2}} \mathrm{~d} g \mathrm{~d} h^{2} \\
& =\int_{\frac{C}{b^{2}}}^{\frac{C}{a^{2}}} \frac{C g^{-2}}{b^{2}-a^{2}}\left(1-e^{\left.-\frac{\gamma N_{0}}{p(a) g}\right) \mathrm{d} g}\right. \\
& =1-\frac{p(a) C}{N_{0}\left(b^{2}-a^{2}\right) \gamma}\left(e^{-\frac{\gamma a^{2} N_{0}}{p(a) C}}-e^{-\frac{\gamma b^{2} N_{0}}{p(a) C}}\right),
\end{aligned}
$$

where we omit the subscribe $m$ since the calculation is based on the distance, instead of number of sensors. Then, considering the SNR threshold $\gamma_{t h}$, the packet loss probability inside the circle ring can be expressed as

$$
F_{\Gamma}\left(\gamma_{t h} \mid a \leq l \leq b\right)=1-\frac{p(a) C\left(e^{-\frac{\gamma_{t h} a^{2} N_{0}}{p(a) C}}-e^{-\frac{\gamma_{t h} b^{2} N_{0}}{p(a) C}}\right)}{N_{0}\left(b^{2}-a^{2}\right) \gamma_{t h}}
$$

For $M_{a}$ sensors with active probability $P_{a}(a)$, we have the packet loss probability as

$$
F_{\Gamma}\left(\gamma_{t h} \mid a \leq l \leq b, M\right)=\left(F_{\Gamma}\left(\gamma_{t h} \mid a \leq l \leq b\right)\right)^{M_{a} P_{a}(a)} .
$$

Furthermore, we assume that the transmission error probability $\varepsilon_{m}$ is constant for each sensor-to-plant link, which is represented as $\varepsilon_{0}$. Then, the $\mathrm{CDF}$ of the overall packet loss probability can be expressed as

$$
F_{\Gamma}\left(\gamma_{t h}\right)=\int_{0}^{R} f_{a}(a)\left(F_{\Gamma}\left(\gamma_{t h} \mid a \leq l \leq b, M\right)\right) \mathrm{d} a,
$$

where $l \rightarrow 0$ and $f_{a}(a)=2 a / R^{2}$. Then, the overall reliability probability for the plant can be expressed as

$$
\operatorname{Pr}\left\{\alpha_{n}=1\right\}=1-\varepsilon_{0}-F_{\Gamma}\left(\gamma_{t h}\right) .
$$

\section{Optimization Problem}

In this subsection, we formulate the optimization problem, which can be described in Problem 0, i.e., $\mathbf{P}_{0}$,

$$
\begin{array}{ll} 
& \mathbf{P}_{0}: \min _{P_{a}(a), p(a)} J \\
\text { s.t. } & \mathbb{E}\left[\Delta\left(\xi_{n+1}\right) \mid \xi_{n}\right] \leq \rho \Delta\left(\xi_{n}\right)+\operatorname{Tr}\left(\mathbf{Q R}^{\prime}\right), \\
& \operatorname{Pr}\left\{\alpha_{n}=1\right\}=1-\varepsilon \geq 1-\varepsilon_{t h}, \\
& 0 \leq P_{a}(a) \leq 1, \\
& 0 \leq p(a) \leq p_{\max },
\end{array}
$$

where $J=M \int_{0}^{R} f_{a}(a) p(a) P_{a}(a) \mathrm{d} a$ is based on the distance between the sensor and plant, and $\operatorname{Pr}\left\{\alpha_{n}=1\right\}=1-\varepsilon=$ $1-\varepsilon_{0}-F_{\Gamma}\left(\gamma_{t h}\right)$. Here, (25a) is the objective function, i.e., power consumption, constrained by communication and control. Given time and frequency resource, power consumption $J$ is equivalent to energy consumption. Equation (25b) is the constraint coming from control convergence rate. Equation (25c) is the reliability constraint coming from URLLC, where $\varepsilon_{t h}$ is the threshold of the packet loss probability in URLLC. Equation (25d) is the constraint on sensors' activation probability at distance $a$, and equation (25e) is the constraint on sensors' transmission power at distance $a$.

In (25), the sensors autonomously decide whether to be activated to participate in the control process. Then, the activated sensors need to adjust their transmission power without intercommunication to minimize total energy consumption, which leads to solving $\mathbf{P}_{0}$ extremely challenging. In the following of this paper, we propose a failed reduction efficiency (FRE) to deal with $\mathbf{P}_{0}$.

\section{The Optimal Solution For D2D ACtivation And TRANSMISSION}

In this section, we solve the optimization problem $\mathbf{P}_{0}$ to obtain the D2D activation and power allocation method. First, we analyze the relationship between communication and control, and convert the control constraint in (25b) into the communication constraint based on the relationship. Then, $\mathbf{P}_{0}$ with communication and control constraints can be recast to a problem that depends only on communication constraints. Finally, each sensor can obtain its optimal activation probability and transmission power by balancing FRE for all sensors based on the distance between the sensors and the plant.

\section{A. Relationship Between Control and Communication}

From (13), we find that the expression $\mathbb{E}\left[\Delta\left(\xi_{n+1}\right) \mid \xi_{n}\right]$ depends on the packet transmission probability, where we can obtain that the Lyapunov-like function can be expressed as

$$
\begin{aligned}
& \mathbb{E}\left[\Delta\left(\xi_{n+1}\right) \mid \xi_{n}\right]=\operatorname{Pr}\left\{\alpha_{n}=1\right\} \xi_{n}^{T} \mathbf{\Omega}_{e_{1}}^{T} \mathbf{Q} \boldsymbol{\Omega}_{e_{1}} \xi_{n} \\
& +\operatorname{Pr}\left\{\alpha_{n}=0\right\} \xi_{n}^{T} \mathbf{\Omega}_{e_{0}}^{T} \mathbf{Q} \boldsymbol{\Omega}_{e_{0}} \xi_{n}+\operatorname{Tr}\left(\mathbf{Q} \mathbf{R}^{\prime}\right) .
\end{aligned}
$$

Submitting (26) into (25b), we can obtain

$$
\operatorname{Pr}\left\{\alpha_{n}=1\right\} \geq \frac{\xi_{n}^{T}\left(\boldsymbol{\Omega}_{e_{0}}^{T} \mathbf{Q} \boldsymbol{\Omega}_{e_{0}}-\rho \mathbf{Q}\right) \xi_{n}}{\xi_{n}^{T}\left(\boldsymbol{\Omega}_{e_{0}}^{T} \mathbf{Q} \boldsymbol{\Omega}_{e_{0}}-\boldsymbol{\Omega}_{e_{1}}^{T} \mathbf{Q} \boldsymbol{\Omega}_{e_{1}}\right) \xi_{n}},
$$


where $\xi_{n} \neq 0$. Let

$$
c=\sup _{y \in \mathbb{R}^{n}, y \neq 0} \frac{y^{T}\left(\mathbf{\Omega}_{e_{0}}^{T} \mathbf{Q} \boldsymbol{\Omega}_{e_{0}}-\rho \mathbf{Q}\right) y}{y^{T}\left(\mathbf{\Omega}_{e_{0}}^{T} \mathbf{Q} \boldsymbol{\Omega}_{e_{0}}-\boldsymbol{\Omega}_{e_{1}}^{T} \mathbf{Q} \boldsymbol{\Omega}_{e_{1}}\right) y}
$$

represent the supremum of the rihgt-hand term in (27). According to [2], we can obtain the optimal $c^{*}$. Then, we can obtain that the communication reliability is not constrained by the requirement in URLLC, but the control reduction rate requirement from control systems, i.e., $c^{*}(\rho)$. Then, $\mathbf{P}_{0}$ can be rewrite as

$$
\begin{array}{ll} 
& \mathbf{P}_{1}: \min _{P_{a}(a), p(a)} J \\
\text { s.t. } & F_{\Gamma}\left(\gamma_{t h}\right) \leq 1-\varepsilon_{0}-c^{*},(25 d), \text { and }(25 e) .
\end{array}
$$

\section{B. Optimal Sensor Activation and Power Allocation}

In this subsection, we propose an optimal algorithm to solve the problem $\mathbf{P}_{1}$. Compared with traditional exhaustive search algorithm with exponential complexity, the complexity of our algorithm grows linearly, which is easy to be deployed and used in real scenarios.

1) Failed Reduction Efficiency: To obtain the optimal solution, we first introduce the FRE. The average energy consumption can be expressed as

$$
J_{c}(a)=P_{a}(a) p(a)
$$

Then, the packet loss probability in (22) can be rewritten as

$$
F_{\Gamma}\left(\gamma_{t h} \mid a \leq l \leq b, M\right)=\left(F_{\Gamma}\left(\gamma_{t h} \mid a \leq l \leq b\right)\right)^{M_{a} \frac{J_{c}(a)}{p(a)}} .
$$

The FRE is defined as the ratio of the packet loss probability and the energy consumption $J_{c}(a)$. Then, FRE can be obtained by taking partial derivation on $J_{c}(a)$ in (31), i.e.,

$$
\begin{aligned}
& D\left(J_{c}(a), p(a), a\right)=\frac{\partial F_{\Gamma}\left(\gamma_{t h} \mid a \leq l \leq b, M\right)}{\partial J_{c}(a)} \\
& =\frac{M_{a}}{p(a)} \ln \left(F_{\Gamma}\left(\gamma_{t h} \mid a \leq l \leq b\right)\right)\left(F_{\Gamma}\left(\gamma_{t h} \mid a \leq l \leq b\right)\right)^{M_{a} \frac{J_{c}(a)}{p(a)}},
\end{aligned}
$$

where the FRE is negative since $\ln \left(F_{\Gamma}\left(\gamma_{t h} \mid a \leq l \leq b\right)\right)$ is negative. This means that lower FRE leads to larger power efficiency. Thus, to minimize transmission power in (25a), we can maintain high power efficiency and omit low power efficiency by FRE adjustment, where we can obtain both active probability and transmission power allocation during this process since FRE is related with the active probability $p(a)$ as shown in (32).

2) Transmission Power Simplification for FRE: Given average power $J_{c}\left(a_{0}\right)$ at a certain distance $a_{0}$, the FRE is a function of $p\left(a_{0}\right)$. Once the optimal $p(a)$ is obtained for all $a$ and $J_{c}(a)$, i.e., $p^{*}(a)=f\left(a, J_{c}(a)\right)$, the FRE is a function of $J_{c}(a)$ and $a$. Then, by $p^{*}(a)=f\left(a, J_{c}(a)\right)$, the parameters to solve (29) reduce from three to two, where $p^{*}(a)$ can be calculated after we obtain optimal $J_{c}(a)$. Next, we focus on obtaining the optimal $p^{*}(a)$ for given $a$ and $J_{c}(a)$, which can be obtain by solving the following optimization problem,

$$
\begin{array}{cc}
\mathbf{P}_{2}: \min _{p(a)} & D\left(J_{c}(a), p(a), a\right) \\
\text { s.t. } & 0 \leq p(a) \leq p_{\max } .
\end{array}
$$

We need to note that solving $\mathbf{P}_{2}$ in (33) is equivalent to solving $\mathbf{P}_{1}$ in (29) for given $a$ and $J_{c}(a)$. Taking partial derivation on $p(a)$ in (33a), we can obtain (34) on the top of next page.

Observing (34), it is difficult to solve (33) by the derivation. To solve the problem, we adopt exhaustive search method in [43] to obtain the optimal $p^{*}(a)$. The complexity of the method is determined by the length of quantized values of $a$ and $J_{c}(a)$. We assume the length of quantized values of $a$ and $J_{c}(a)$ is $\mu$ and $v$, respectively. Then, the computing complexity is $\mu \times v$.

3) Optimal Solution: By obtaining $p^{*}(a)=f\left(a, J_{c}(a)\right)$, the parameters to solve (29) reduce from three to two. Then, the FRE can be expressed as $D\left(J_{c}(a), p^{*}(a), a\right)$. To obtain the solution for (29), we can prove that the following property holds.

Property 1: For each distance a, the FRE with $p^{*}(a)$ monotonically increases with average power $J_{c}(a)$.

Proof: See Appendix A.

This property indicates that larger energy consumption at the sensor leads to lower power efficiency, where we can obtain the minimum FRE when $J_{c}(a)=0$. We can set a FRE threshold $\varphi<0$ to determine the transmission power of the sensor. Then, we have the following property about the threshold.

Property 2: The threshold $\varphi$ is suitable for all sensors inside the circle with radius $R$.

Proof: See Appendix B.

By Property 2, we can obtain $J_{c}(a)$ as

$\begin{cases}J_{c}(a)=0, & \text { if } D\left(0, p^{*}(a), a\right)>\varphi \\ J_{c}(a)>0 \text { and } D\left(J_{c}(a), p^{*}(a), a\right)=\varphi, & \text { if } D\left(0, p^{*}(a), a\right) \leq \varphi\end{cases}$

Substituting (35) into (32), we can obtain the optimal $J_{c}^{*}(a)$ as

$$
\begin{aligned}
& J_{c}^{*}(a)=\max \left(0, \frac{p^{*}(a)}{M_{a}} .\right. \\
& \left.\frac{\ln \left(-\varphi p^{*}(a)\right)-\ln \left(-M_{a} \ln \left(F_{\Gamma}\left(\gamma_{t h} \mid a \leq l \leq b\right)\right)\right.}{\ln \left(F_{\Gamma}\left(\gamma_{t h} \mid a \leq l \leq b\right)\right)}\right) .
\end{aligned}
$$

Then, substituting (36) into (31), we can obtain the failed probability for distance $a$ as

$$
F_{\Gamma}\left(\gamma_{t h} \mid a \leq l \leq b, M, \varphi\right)=\left(F_{\Gamma}\left(\gamma_{t h} \mid a \leq l \leq b\right)\right)^{M_{a} \frac{J_{c}^{*}(a)}{p^{*}(a)}}
$$

From (24) and (37), we can obtain the reliability as

$$
\operatorname{Pr}^{*}\left\{\alpha_{n}=1\right\}=1-\varepsilon_{0}-\int_{0}^{R} f_{a}(a) F_{\Gamma}\left(\gamma_{t h} \mid a \leq l \leq b, M, \varphi\right) \mathrm{d} a .
$$

Let $\operatorname{Pr}^{*}\left\{\alpha_{n}=1\right\}=c^{*}$. Then, we can obtain the optimal $J_{c}^{*}(a)$ for the sensors by finding suitable $\varphi$. Finally, the transmission power $p^{*}(a)$ for the sensor and the activate probability $P_{a}^{*}(a)$ 


$$
\begin{aligned}
& D^{\prime}\left(J_{c}(a), p(a), a\right)=\frac{\partial D\left(J_{c}(a), p(a), a\right)}{\partial p(a)}=\frac{M_{a}\left(F_{\Gamma}\left(\gamma_{t h} \mid a \leq l \leq b\right)\right)^{M_{a} \frac{J_{c}(a)}{p(a)}}}{p^{3}(a)\left(F_{\Gamma}\left(\gamma_{t h} \mid a \leq l \leq b\right)\right)} \ln \left(F_{\Gamma}\left(\gamma_{t h} \mid a \leq l \leq b\right)\right) . \\
& {\left[J_{c}(a) M_{a} \ln \left(F_{\Gamma}\left(\gamma_{t h} \mid a \leq l \leq b\right)\right)+1\right]\left[p(a)\left(F_{\Gamma}\left(\gamma_{t h} \mid a \leq l \leq b\right)\right)^{\prime}-\left(F_{\Gamma}\left(\gamma_{t h} \mid a \leq l \leq b\right)\right) .\right.} \\
& \left.\ln \left(F_{\Gamma}\left(\gamma_{t h} \mid a \leq l \leq b\right)\right)\right] .
\end{aligned}
$$

where $\left(F_{\Gamma}\left(\gamma_{t h} \mid a \leq l \leq b\right)\right)^{\prime}$ is the partial derivation on $p(a)$.

can be obtained by solving $p^{*}(a)=f\left(a, J_{c}(a)\right)$ and (30), respectively.

Finally, we briefly summary the above iterative method. After the constrain on control convergence rate converted to the constraint on communication reliability, the original problem $P_{0}$ can be rewritten as a pure communication optimization problem $P_{1}$ for given convergence rate, where the parameters are distance $a$, activation probability $P(a)$, and transmission power $p(a)$. By defining FRE, $P_{1}$ can be further rewritten as $P_{2}$. We rewrite average transmission power as the product of activation probability and transmission power at distance $a$, i.e., $J_{c}(a)=P_{a}(a) p(a)$. Then, the optimal $J_{c}^{*}(a)$ can be obtained by minimizing FRE, where (36) is the closed-form expression for $J_{c}^{*}(a)$. What follows, the optimal transmission power $p^{*}(a)$ and activation probability $P_{a}^{*}(a)$ can be obtained by solving equations, i.e., $p^{*}(a)=f\left(a, J_{c}(a)\right)$ and (30) for each distance $a$. Finally, by iterating distance $a$, we can obtain optimal $p^{*}(a)$ and $P_{a}^{*}(a)$ for each $a$.

4) Computing Complexity: From the above discussion, the computing complexity is $\mu \times v$ with respect to time by exhaustive search method to find $p^{*}(a)=f\left(a, J_{c}(a)\right)$. If the computing complexity in finding optimal solution is $\theta$ with respect to time. Then, the total computing complexity is $\eta=\theta \times \mu \times v$ with respect to time. Thus, the computing complexity of the proposed method is $O(\eta)$ and increases linearly with respect to time.

\section{Simulation Results}

In this section, we provide simulation results to demonstrate the performance of the proposed method, where the system model is the same as shown in Fig. 1.

For URLLC, we assume that the bandwidth is $1 \mathrm{MHz}$, the single-sided noise spectral density is $-174 \mathrm{dBm} / \mathrm{Hz}$, the largescale path loss constants is $C=-113.4 \mathrm{~dB}$, the radius of the circle is $R=100 \mathrm{~m}$, the number of sensors is $M=200$, the maximum transmission power for the sensors is $-17 \mathrm{dBm}$, the transmission error probability is $\varepsilon_{0}=10^{-6}$, and the transmission time delay is $T_{u}=0.5 \mathrm{~ms}$. In addition, the SNR thresholds are $\left[\begin{array}{ll}5 & 10\end{array}\right] \mathrm{dB}$. In addition, since traditional cellular assisted method and autonomous method can not guarantee the QoS requirement in URLLC, we consider a method that all the sensors are activated to guarantee the URLLC requirement as the traditional D2D transmission method for comparison.

For control, we assume that $\Omega_{e_{0}}=2.5$ and $\Omega_{e_{1}}=0.8$ and the given positive definite weight matrix is $\mathbf{Q}=1$, the variance of the disturbance matrix $\mathbf{n}_{m}(t)$ is 1 , i.e., $\mathbf{R}_{m}=1$, and the sample period is $s_{m, n}=100 \mathrm{~ms}$. In addition, we adopt the average

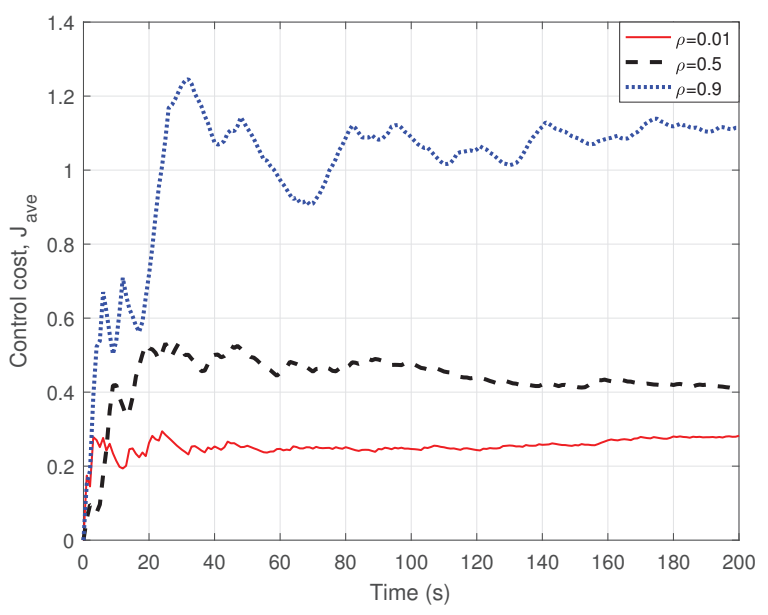

Fig. 4: Control cost with time increasing.

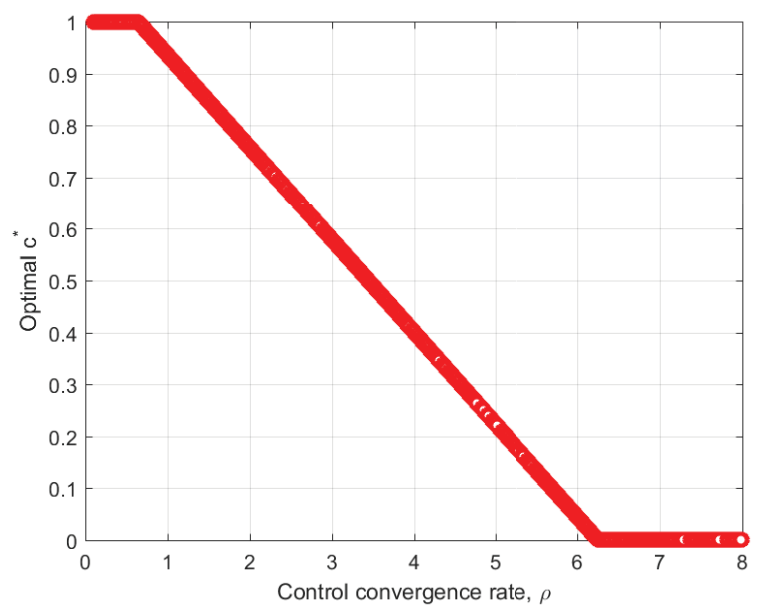

Fig. 5: Optimal $c^{*}$ with different control convergence rate $\rho$.

control cost to evaluate the control performance and can be expressed as [44]

$$
J_{\text {ave }}=\frac{\sum_{n=1}^{N} x_{n}^{2}}{N}
$$

where $N=T / s_{m, n}$ is adopted, and $T$ is the total time of the control process.

\section{A. Control performance}

Fig. 4 shows the control cost $J_{\text {ave }}$ with time increasing, where different control convergence rates $\rho$ are considered, 


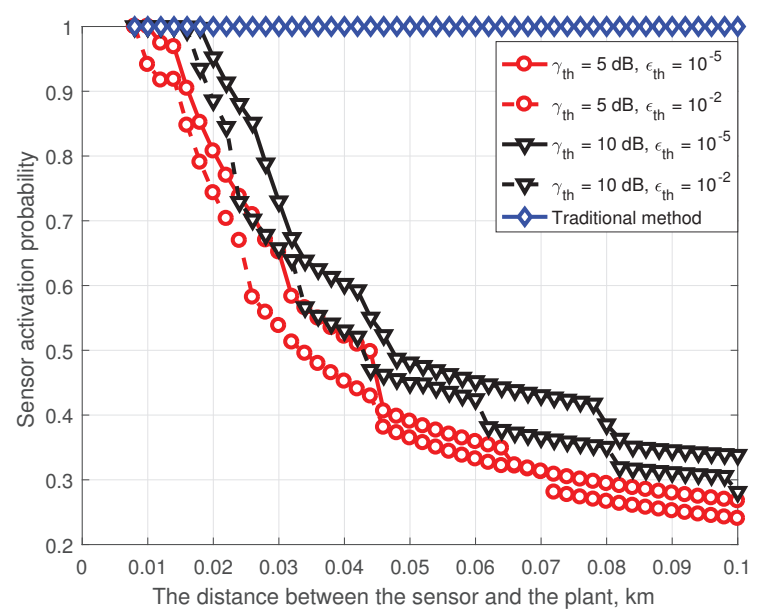

Fig. 6: The sensor activation probability with different distance between the sensor and the plant.

i.e., 0.01, 0.5, and 0.9. From the figure, all the curves increase at the initial time. This is because the changes of plant state leads to the increasing of $J_{\text {ave }}$ before the control system being stable. Furthermore, with time increasing, the control state will be stable and the control cost curves maintains in a horizontal level, where the control cost increases when control convergence rates $\rho$ increases. This indicates small $\rho$ leads to low the average control cost.

Fig. 5 shows the constraint of the control on the communication, i..e, the optimal $c^{*}$, when control convergence rate $\rho$ is different. From the figure, the curve decreases monotonically from $c^{*}=1$ to $c^{*}=0$ with $\rho$, which matches the expression in (28), i.e., $c^{*}$ decreases monotonically with respect to $\rho$. Thus, higher communication reliability guarantees lower $\rho$, which leads to lower control cost, i.e, higher control performance. This is reasonable since higher communication reliability can maintain fast and steady state update, which can reduce the control cost. On the contrary, lower communication reliability results in more packet loss, which can violate the required control convergence rate and then leads to higher control cost [2]. Since the communication reliability cannot be larger than 1 and less than $0, c^{*}$ remains 0 when the control convergence rate $\rho$ is larger than 6.2 .

\section{B. Communication performance}

Fig. 6 shows sensor activation probability when the distance between the sensor and the plant is different, where we considered different SNR threshold $\gamma_{t h}=5 \mathrm{~dB}$ and $\gamma_{t h}=10$ $\mathrm{dB}$. In addition, we asume that $\varepsilon_{t h}=\varepsilon_{0}-c^{*}$, where different reliability requirements are considered, i.e., $\varepsilon_{t h}=10^{-2}$ and $\varepsilon_{t h}=10^{-5}$. From the figure, all curves decrease from 1 with distance increasing. This is reasonable since small distance between sensor and plant leads to that small transmission power can guarantee the SNR threshold with large probability. Considering different SNR thresholds $\gamma_{t h}$, the curve with large $\gamma_{t h}$ is higher than that with small $\gamma_{t h}$ for given distance and given $\varepsilon_{t h}$. This is reasonable since more active sensors are needed to satisfy larger $\gamma_{t h}$. Thus, the activation probability

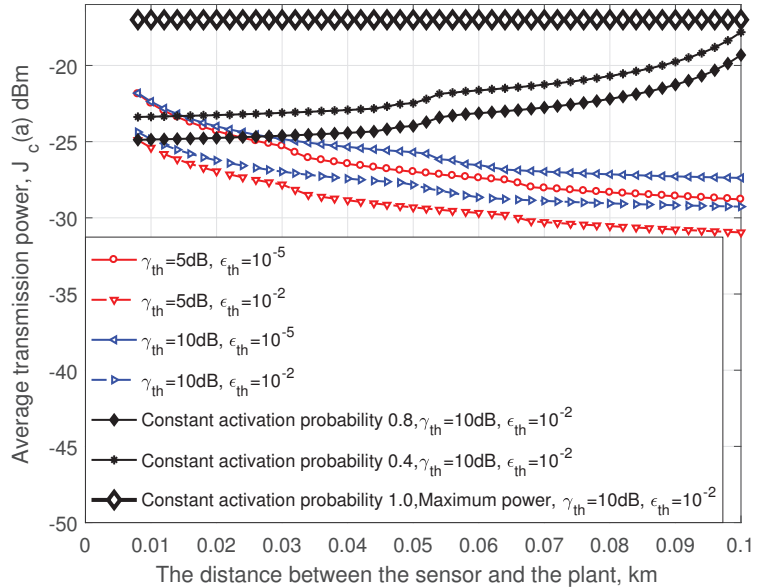

Fig. 7: The average transmission power with different distance between the sensor and the plant.

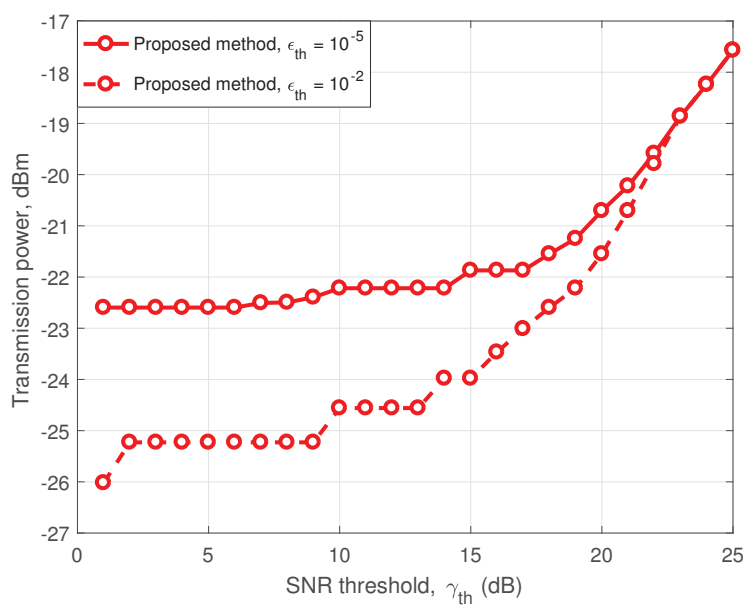

Fig. 8: The transmission power allocation with different SNR threshold $\gamma_{t h}$.

is larger for larger $\gamma_{t h}$. Moreover, considering different $\varepsilon_{t h}$, the curve with small $\varepsilon_{t h}$ is higher than that with large $\varepsilon_{t h}$ for given distance and given $\gamma_{t h}$. This is reasonable since high active probability can guarantee high reliability, i.e., small $\varepsilon_{t h}$. In addition, compared with the traditional method with activation probability being 1 , the proposed method in this paper do not need all sensors keeping active. Furthermore, from Fig. 6, we can observe that when the distance is large, the activation probability becomes small. This means that more sensors are required to achieve the required reliability if sensors are deployed from a large distance. In other words, the relationship between reliability and the number of sensors is related to the distance.

Fig. 7 shows the average transmission power $J_{c}(a)$ when the distance between the sensor and the plant is different, where the SNR threshold $\gamma_{t h}$ and packet loss threshold $\varepsilon_{t h}$ are the same with Fig. 6. From the figure, all the curves of the proposed method decrease with distance increasing. This is reasonable since fewer sensors are activated when the distance 
is larger as shown in Fig. 6. Thus, the average transmission power $J_{c}(a)$ is lower at larger distance. Considering different SNR thresholds $\gamma_{t h}$, the curve with large $\gamma_{t h}$ is high than that with small $\gamma_{t h}$ for given distance and given $\varepsilon_{t h}$. This is reasonable since more transmission power is needed to satisfy larger $\gamma_{t h}$. Moreover, considering different $\varepsilon_{t h}$, the curve with small $\varepsilon_{t h}$ is higher than that with large $\varepsilon_{t h}$ for given distance and given $\gamma_{t h}$. This is reasonable since more transmission power can guarantee high reliability, i.e., small $\varepsilon_{t h}$. Moreover, compared with traditional method with maximum transmission power to maintain URLLC and control requirements, the average transmission power of the proposed is significantly lower, e.g., when $\gamma_{t h}=5 \mathrm{~dB}$ and $\varepsilon_{t h}=10^{-5}$, the transmission power the proposed method is reduced by about $14 \mathrm{~dB}$ at distance $100 \mathrm{~m}$.

Furthermore, we also consider the case when the activation probability is considered with $\gamma_{t h}=10 \mathrm{~dB}$ and $\epsilon_{t h}=10^{-2}$, where only communication reliability from URLLC is considered. Both the curves with activation probabilities 0.4 and 0.8 monotonously increase with distance. This is because the activated sensors should try to maintain the reliability requirement with more transmission power when the distance is larger, which leads to the larger average power, i.e., $J_{c}(a)=$ $P_{a}(a) p(a)$ in (29) at larger distance. From the figure, the average power consumption of the proposed method optimizing activation probability is significantly lower than that with the same probability. For instance, the average transmission power of the proposed method is reduced by about $100 \% \mathrm{~dB}$ compared with the case that activation probability is $40 \%$ and only URLLC is considered when $\gamma_{t h}=10 \mathrm{~dB}$ and $\epsilon_{t h}=10^{-2}$.

Fig. 8 indicates the transmission power allocation when SNR thresholds are different, where the distance between the sensor and the plant is $0.02 \mathrm{~km}$. In addition, we consider different $\varepsilon_{t h}$. From the figure, both the curves of the proposed method increases with SNR threshold $\gamma_{t h}$ increasing. This is reasonable since more transmission power at the sensor is needed to guarantee larger SNR threshold $\gamma_{t h}$. Considering different packet loss threshold $\varepsilon_{t h}$, large $\varepsilon_{t h}$ leads to larger transmission power, which is apparent. However, the traditional method need to transmit with maximum available power to maintain larger SNR threshold $\gamma_{t h}$ with no information about the plant. From the figure, the proposed method can reduce the energy consumption by at most $9 \mathrm{~dB}$ compared with the traditional method when SNR threshold is $\gamma_{t h}=1$ $\mathrm{dB}$ and the packet loss threshold is $\varepsilon_{t h}=10^{-2}$.

\section{CONCLusions}

In this paper, we proposed an autonomous D2D transmission method in URLLC for real-time wireless control systems, where both reliability requirement in URLLC and control requirement were jointly considered. In particular, we formulated an optimization problem to minimize transmission energy consumption under the constraints of communication reliability and control convergence rate. To solve the problem, we first discussed the relationship between control convergence rate and communication reliability, where the control convergence rate constraint was converted into the constraint on communication reliability. Then, we proposed a probability-based D2D activation method, where we set a threshold to determine the transmission strategy of the sensors. This allowed each sensor autonomously to decide whether to participate in the control process with guaranteed reliability requirement in URLLC and control convergence rate requirement, which can significantly reduce the energy consumption compared with the traditional D2D transmission method.

\section{APPENDIX A}

This appendix provides the proof for Property 1, i.e., the FRE with $p^{*}(a)$ monotonically increases with average power $J_{c}(a)$ for each distance $a$.

We assume two average power $J_{c}^{i}(a)$ and $J_{c}^{j}(a)$ for distance $a$, where $J_{c}^{i}(a)<J_{c}^{j}(a)$. Then, we have

$$
\begin{aligned}
& D\left(J_{c}^{j}(a), p(a), a\right) \\
& =\frac{M_{a}}{p(a)} \ln \left(F_{\Gamma}\left(\gamma_{t h} \mid a \leq l \leq b\right)\right)\left(F_{\Gamma}\left(\gamma_{t h} \mid a \leq l \leq b\right)\right)^{M_{a} \frac{J_{c}^{j}(a)}{p(a)}} \\
& =D\left(J_{c}^{i}(a), p(a), a\right)\left(F_{\Gamma}\left(\gamma_{t h} \mid a \leq l \leq b\right)\right)^{M_{a} \frac{J_{c}^{j}(a)-J_{c}^{i}(a)}{p(a)}} .
\end{aligned}
$$

Then, for optimal $p^{*}(a)$, we have $D\left(J_{c}^{i}(a), p^{*}(a), a\right) \leq$ $D\left(J_{c}^{i}(a), p(a), a\right)$. Since $0 \leq\left(F_{\Gamma}\left(\gamma_{t h} \mid a \leq l \leq b\right)\right) \leq 1$, then we can obtain

$$
\begin{aligned}
& D\left(J_{c}^{j}(a), p(a), a\right) \\
& =D\left(J_{c}^{i}(a), p(a), a\right)\left(F_{\Gamma}\left(\gamma_{t h} \mid a \leq l \leq b\right)\right)^{M_{a} \frac{J_{c}^{j}(a)-J_{c}^{i}(a)}{p(a)}} \\
& \geq D\left(J_{c}^{i}(a), p^{*}(a), a\right)\left(F_{\Gamma}\left(\gamma_{t h} \mid a \leq l \leq b\right)\right)^{M_{a} \frac{J_{c}^{j}(a)-J_{c}^{i}(a)}{p(a)}} \\
& \geq D\left(J_{c}^{i}(a), p^{*}(a), a\right),
\end{aligned}
$$

which indicates that the FRE with $p^{*}(a)$ monotonically increases with average power $J_{c}(a)$ for each distance $a$.

\section{APPENDIX B}

This appendix provides the proof for Property 2, i.e., the threshold $\varphi$ is suitable for all sensors inside the circle with radius $R$.

For the $m$-th sensor with distance $l_{m}$ from the plant, we set a threshold $\varphi_{m}$. Then, (35) can be rewritten as (42) on the top of next page.

We assume that

$$
\left\{\begin{array}{l}
\varphi_{m} \leq \varphi, m=1,2, \cdots, Q \\
\varphi_{m}>\varphi, m=Q+1, Q+2, \cdots, M .
\end{array}\right.
$$

Then, we have the CDF of the overall packet loss probability as

$$
F_{\Gamma}\left(\gamma_{t h}\right)=\prod_{m=1}^{M} F_{\Gamma}\left(\gamma_{t h}, \varphi_{m}\right)=1-\varepsilon_{0}-c^{*},
$$

where $F_{\Gamma}\left(\gamma_{t h}, \varphi_{m}\right)$ is the overall packet loss probability of the $m$-th sensor with threshold $\varphi_{m}$. In addition, to obtain minimum transmission power, $F_{\Gamma}\left(\gamma_{t h}\right)=1-\varepsilon_{0}-c^{*}$ should hold. The total average transmission power can be expressed as

$$
J_{c}=\sum_{m=1}^{M} J_{c}\left(a_{m}, \varphi_{m}\right) .
$$




$$
\left\{\begin{array}{lr}
J_{c}\left(a_{m}\right)=0, & \text { if } D\left(0, p^{*}\left(a_{m}\right), a_{m}\right)>\varphi_{m}, \\
J_{c}\left(a_{m}\right)>0 \text { and } D\left(J_{c}\left(a_{m}\right), p^{*}\left(a_{m}\right), a_{m}\right)=\varphi_{m}, & \text { if } D\left(0, p^{*}\left(a_{m}\right), a_{m}\right) \leq \varphi_{m} .
\end{array}\right.
$$

If $\varphi_{m}=\varphi$, we have

$$
F_{\Gamma}\left(\gamma_{t h}\right)=\prod_{m=1}^{M} F_{\Gamma}\left(\gamma_{t h}, \varphi\right)=1-\varepsilon_{0}-c^{*}
$$

Then, the difference between (44) and (46) can be expressed as

$$
\begin{aligned}
0 & =\prod_{m=1}^{M} F_{\Gamma}\left(\gamma_{t h}, \varphi\right)-\prod_{m=1}^{M} F_{\Gamma}\left(\gamma_{t h}, \varphi_{m}\right) \\
& =-\int_{J_{c}\left(a_{m}, \varphi_{m}\right)}^{J_{c}\left(a_{m}, \varphi\right)} D\left(J_{c}(a), p^{*}(a), a\right) \mathrm{d}\left(J_{c}(a)\right) \\
& \leq \varphi\left(J_{c}\left(a_{m}, \varphi_{m}\right)-J_{c}\left(a_{m}, \varphi\right)\right) .
\end{aligned}
$$

Then, we have

$$
J_{c}\left(a_{m}, \varphi_{m}\right) \geq J_{c}\left(a_{m}, \varphi\right),
$$

which means that the threshold $\varphi$ is suitable for all sensors to obtain minimum transmission power inside the circle with radius $R$.

\section{REFERENCES}

[1] M. Zhan, Z. Pang, M. Xiao, M. Luvisotto and D. Dzung, "Wireless highperformance communications: improving effectiveness and creating ultrahigh reliability with channel coding," IEEE Indus. Elec. Mag., vol. 12, no. 3, pp. 32-37, Sept. 2018

[2] B. Chang, L. Zhang, L. Li, G. Zhao, and Zhi Chen, "Optimizing resource allocation in URLLC for real-time wireless control systems," IEEE Trans. Veh. Tech., vol. 68, no, 9, pp. 8916-8927, Sept. 2019.

[3] G. Zhao, M. A. Imran, Z. Pang, Z. Chen, and Liying Li, "Toward real-time control in future wireless networks: communication-control co-design," IEEE Commun. Mag., vol.57, no. 2, pp. 138-144, Feb. 2019.

[4] 3GPP, "3GPP Release 16," Jul. 2018, [Online]. Available: http: //www.3gpp.org/release-16

[5] M. Ahmed, Y. Li, M. Waqas, M. Sheraz, D. Jin, and Zhu Han, "A survey on socially aware device-to-device communications," IEEE Commun. Surveys Tut., vol. 20, no. 3, pp. 2169-2197, 3rd Quart., 2018.

[6] F. Jameel, Z. Hamid, F. Jabeen, S. Zeadally, and M. Javed, "A survey of device-to-device communications: research issues and challenges," IEEE Commun. Surveys Tut., vol. 20, no. 3, pp. 2133-2168, 3rd Quart., 2018.

[7] R. Ansari et al., "5G D2D networks: techniques, challenges, and future prospects," IEEE Syst. J., vol. 12, no. 4, pp. 3970-3984, Dec. 2018.

[8] B. Chang, G. Zhao, Z. Chen, P. Li, and L. Li, "D2D transmission scheme in URLLC enabled real-time wireless control systems for tactile internet," IEEE Global Communications Conference (GLOBECOM), Dec. 2019, pp. $1-6$.

[9] K. J. Zou et al., "Proximity discovery for device-to-device communications over a cellular network," IEEE Commun. Mag., vol. 52, no. 6, pp. 98-107, Jun. 2014.

[10] A. Asadi, Q. Wang, and V. Mancuso, "A survey on device-to-device communication in cellular networks," IEEE Commun. Surveys Tut., vol. 16, no. 4, pp. 1801-1819, 4th Quart., 2018.

[11] P. Mach, Z. Becvar, and T. Vanek, "In-band device-to-device communication in OFDMA cellular networks: a survey and challenges," IEEE Commun. Surveys Tut., vol. 17, no. 4, pp. 1885-1922, 4th Quart., 2015.

[12] N. Nguyen, K. Choi, L. Song, and Z. Han, "ROOMMATEs: an unsupervised indoor peer discovery approach for LTE D2D communications," IEEE Trans. Veh. Tech., vol. 67 , no. 6, pp. 5069-5083, Jun. 2018.

[13] D. Burghal, A. Tehrani, and A. Molisch, "On expected neighbor discovery time with prior information: modeling, bounds and optimization," IEEE Trans. Wireless Commun., vol. 17, no. 1, pp. 3124-3134, Dec. 2014.

[14] B. Chang, G. Zhao, M. Imran, L. Li, and Z. Chen, "Dynamic wireless QoS analysis for real-time control in URLLC," IEEE Globecom Workshops (GC Wkshps), Dec. 2018, pp. 1-4.
[15] L. Schenato, B. Sinopoli, M. Franceschetti, K. Poola, and S. Sastry, "Foundations of control and estimation over lossy networks," IEEE Proc., vol. 95, no. 1, pp. 163-187, Jan. 2007.

[16] P. Park, J. Araújo, and K. H. Johansson, "Wireless networked control system co-design," IEEE Inter. Conf. Networking, Sensing and Control (ICNSC), 2011, pp.486-491.

[17] P. Park, S. Ergen, C. Fischione, C. Lu, and K. Johansson, "Wireless network design for control systems: a survey," IEEE Commun. Surveys Tut., pp. 1-1, Dec. 2017.

[18] S. Kartakis, A. Fu, M. Mazo, and J. McCann, "Communication schemes for centralized and decentralized event-triggered control systems," IEEE Trans. Control Syst. Technol., vol. 26, no. 6, pp. 2035-2048, Nov. 2018.

[19] R. Mansano, R. Rodrigues, E. Godoy, and D. Colon, "A new adaptive controller in wireless networked control systems: developing a robust and effective controller for energy efficiency," IEEE Indus. App. Mag., vol. 25, no. 2, pp. 12-22, Mar. 2019.

[20] L. Su and G. Chesi, "On the robust stability of uncertain discrete-time networked control systems over fading channels," American Control Conf. (ACC), Jul. 2015, pp. 6010-6015.

[21] C. Peng, D. Yue, and M. Fei, "A higher energy-efficient sampling scheme for networked control systems over IEEE 802.15.4 Wireless Networks," IEEE Trans. Indus. Informat., vol. 12, no. 5, pp. 1766-1774, Oct. 2016.

[22] R. S. Wagner and R. J. Barton, "Performance comparison of wireless sensor network standard protocols in an aerospace environment: ISA100.11a and ZigBee Pro," in Proc. IEEE Aerosp. Conf., 2012, pp. $1-14$.

[23] B. Chang, G. Zhao, L. Zhang, M. A. Imran, and Z. Chen, and L. $\mathrm{Li}$, "Dynamic communication QoS design for real-time wireless control systems," IEEE Sensors J., vol. 20, no, 6, pp. 3005-3015, Mar. 2020.

[24] B. Chang, G. Zhao, Z. Chen, L. Li, and M. A. Imran, "Packet-drop design in URLLC for real-time wireless control systems," IEEE Access, vol. 7, no. 1, pp. 183081-183090, Jul. 2019.

[25] T. Zeng, O. Semiari, W. Saad, and M. Bennis, "Joint communication and control for wireless autonomous vehicular platoon systems," IEEE Trans. Commun., vol. 67, no.11, pp. 7907-7922, Nov. 2019.

[26] T. Zeng, M. Mozaffari, O. Semiari, W. Saad, and M. Debbah, "Wireless communications and control for swarms of cellular-connected UAVs," 2018 52nd Asilomar Conf. Signals, Systems, and Computers, Oct. 2018, pp. 1-5.

[27] K. Gatsis, H. Hassani, and G. J. Pappas, "Latency-reliability tradeoffs for state estimation," IEEE Trans. Automatic Control, pp. 1-1, May 2020.

[28] L. Liu and W. Yu, "A D2D-based protocol for ultra-reliable wireless communications for industrial automation," IEEE Trans. Wireless Commun., vol. 17, no. 8, pp. 9960-9971, Aug. 2018.

[29] Y. Wu, D. Wu, L. Ao, L. Yang, and Q. Fu, "Contention-based radio resource management for URLLC-oriented D2D communications," IEEE Trans. Veh. Tech., vol. 69, no. 9, pp. 5045-5058, Sept. 2020.

[30] I. F. Akyildiz and M. C. Varun, Wireless Sensor Networks, Wiley, New York, 2010.

[31] C. Chen, L. Lyu, S. Zhu, and X. Guan, "On-demand transmission for edge-assisted remote control in industrial network systems," IEEE Trans. Indus. Infor, vol. 16, no. 7, pp. 4842-4854, Jul. 2020.

[32] T. Rappaport and C. McGillem, "UHF fading in factories," IEEE $J$. Select. Areas Commun., vol. 7, no.1, pp. 40-48, Jan. 1989.

[33] A. Azab, H. ElMaraghy, P. Nyhuis, J. Frauenhofer, and M. Schmidt, "Mechanics of change: A framework to reconfigure manufacturing systems," CIRP J. Manufacturing Science and Tech., vol. 6, no. 2, pp. 110119, Jan. 2013.

[34] 3GPP, Study on Scenarios and Requirements for Next Generation Access Technologies. Technical Specification Group Radio Access Network, Technical Report 38.913, Release 14, Oct. 2016.

[35] C. She, C. Yang, and T. Quek, "Cross-layer optimization for ultrareliable and low-latency radio access networks," IEEE Trans. Wireless Commun., vol. 17, no. 1, pp. 127-141, Jan. 2018.

[36] W. Yang, G. Durisi, T. Koch, and Y. Polyanskiy, "Quasi-static multipleantenna fading channels at finite blocklength," IEEE Trans. Inf. Theory, vol. 60, no. 7, pp. 4232-4264, Jul. 2014

[37] G. Durisi, T. Koch, and P. Popovski, "Toward massive, ultrareliable, and low-latency wireless communication with short packets," IEEE Proc., vol.104, no. 9, pp. 1711-1726, Aug. 2016. 
[38] Z. Bubnicki, Modern Control Theory, Springer-Verlag, 2005.

[39] S. Cai and V. Lau, "Zero MAC latency sensor networking for cyberphysical systems," IEEE Trans. Signal Process., vol. 66, no. 14, pp. 38143823, Jul. 2018.

[40] K. Gatsis, A. Ribeiro, and G. Pappas, "State-based communication design for wireless control systems," IEEE 55th Conf. Decision and Control (CDC), Dec. 2016, pp. 129-134.

[41] Z. Fei, B. Li, S. Yang, C. Xing, H. Chen, and L. Hanzo, "A survey of multi-objective optimization in wireless sensor networks: metrics, algorithms, and open problems," IEEE Commun. Surveys Tut., vol. 19, no. 1, pp. 550-586, 1st Quart., 2017.

[42] K. Gatsis, M. Pajic, A. Ribeiro, and G. J. Pappas, "Opportunistic control over shared wireless channels," IEEE Trans. Auto. Control, vol. 60, no. 12, pp. 3140-3155, Dec. 2015.

[43] T. Cormen, C. Leiserson, and R. Rivest, Introduction to Algorithms, Mit Press, Cambridge, 1990

[44] J. Sarangapani and H. Xu, Optimal networked control systems with MATLAB (automation and control engineering), CRC Press, Florida, 2015.

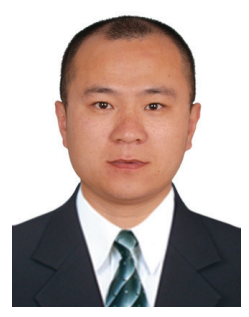

Bo Chang received his B.E., M.E., and Ph.D. Degrees from University of Electronic Science and Technology of China (UESTC) in 2013, 2016, and 2020. From May 2019 to May 2020, he was a visiting student at University of Glasgow, UK. Since August 2020, he has been with the National Key Lab. on Communications, UESTC, where he is currently a Research Fellow. His research interests include ultra-reliable and low-latency communications, communication and control co-design for Introl of robotics in $5 \mathrm{G} / 6 \mathrm{G}$ dustrial Internet-of-Things (IIoT), and remote con-

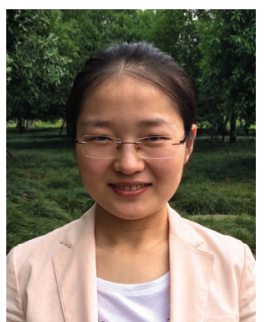

Liying (Emma) Li received her Ph.D. Degrees in Electrical Engineering from University of Electronic Science and Technology of China (UESTC) in 2011. She visited Lehigh University, PA, USA, in 2016. In 2011-2019, she worked as an assistant/associate professor with the School of Automation Engineering, University of Electronic Science and Technology of China (UESTC). Now she is a senior lecturer at Northumbria University Newcastle, UK. Her current research interests are within the areas of $5 \mathrm{G}$ and $6 \mathrm{G}$ communications, in particular high performance and secure remote robotic control.

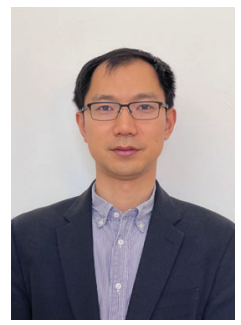

Guodong (Philip) Zhao (SM'16) received his Ph.D. degree in Electrical Engineering from Beihang University, Beijing, China, in 2011. In 2011-2018, he was an associate professor at University of Electronic Science and Technology of China (UESTC), Chengdu, China. In 2018, he joined University of Glasgow in UK as a lecturer (assistant professor). He is an IEEE Senior member. He published 1 authored book in Springer, 1 edited book in MDPI, and 2 book chapters in Wiley. He published more than 100 peer-reviewed research papers (including 20+ IEEE journals), had 2000+ citations at Google Scholar, and won the Best PhD Thesis Award from Beihang University in 2012, a best paper award in IEEE Globecom 2012, and a best poster award in IEEE WCNC 2018. His current research interest is the joint design of wireless communication and control for secure and trustworthy cyber physical systems, with the applications of tele-robotics in $5 \mathrm{G} / 6 \mathrm{G}$, Industry 4.0 , human-robot interaction, autonomous driving, etc.

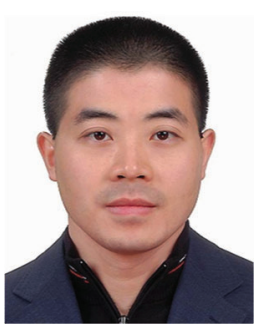

Zhi Chen (SM'16) received B.E., M.E., and Ph.D. degrees in electrical engineering from the University of Electronic Science and Technology of China (UESTC), in 1997, 2000, and 2006, respectively. On April 2006, he joined the National Key Lab of Science and Technology on Communications (NCL), UESTC, and worked as a Professor with this lab from August 2013. He was a Visiting Scholar with the University of California, Riverside during 2010-2011. He is also the Deputy Director of Key Laboratory of Terahertz Technology, Ministry of Education. His current research interests include Terahertz communication, 5G mobile communications and tactile internet.

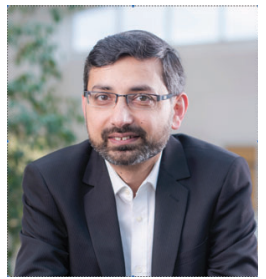

Muhammad Ali Imran (M'03, SM'12) Fellow IET, Senior Member IEEE, Senior Fellow HEA is Dean University of Glasgow UESTC and a Professor of Wireless Communication Systems with research interests in self organised networks, wireless networked control systems and the wireless sensor systems. He heads the Communications, Sensing and Imaging (CSI) research group at University of Glasgow and is Director of Centre for Educational Development and Innovation. He is an Affiliate Professor at the University of Oklahoma, USA; Adjunct Research Professor at Ajman University, UAE and a visiting Professor at 5G Innovation Centre, University of Surrey, UK. He has over 20 years of combined academic and industry experience with several leading roles in multi-million pounds funded projects. He has filed 15 patents; has authored/coauthored over 400 journal and conference publications; has authored 2 books, edited 8 books and authored more than 30 book chapters; has successfully supervised over 40 postgraduate students at Doctoral level. He has been a consultant to international projects and local companies in the area of selforganised networks. 